\title{
Effectiveness and safety of pneumothorax management in newborns without chest-tube insertion
}

\author{
Anna Menshykova, Dmytro Dobryanskyy \\ Department of Paediatrics No. 2, Danylo Halytsky Lviv National Medical University, Lviv, Ukraine
}

\section{ABSTRACT}

Introduction: Pneumothorax is a serious complication associated with increased morbidity and mortality in newborns. However, chest-tube insertion, which is recommended for treatment of this pathology, is an invasive procedure, which can also lead to adverse clinical consequences.

Aim of the study: The aim of this study was to identify clinical features of infants with pneumothorax treated without chest-tube insertion.

Material and methods: In this case series study we report the clinical characteristics and outcomes in 31 outborn infants with pneumothorax, who were successfully managed without chest-tube insertion.

Results: Pneumothorax occurred at the median age of 2 (range: $0-4$ ) days. Ten (32\%) infants had a tension pneumothorax, and in five (16\%) cases bilateral pneumothorax was observed. Mean birth weight of the newborns was $2287.42 \pm 755.45 \mathrm{~g}$ and gestational age $-34.29 \pm 3.2$ weeks. The majority of infants were late preterm (77\%). Surfactant therapy was used in $23 \%$ of all cases. All infants had an underlying primary lung disease. Twenty-three (74\%) infants were on non-invasive respiratory support at the time of pneumothorax occurrence, and 18 (78\%) of them were switched to mechanical ventilation (MV) as soon as the diagnosis was confirmed. CPAP was the most commonly used type of initial respiratory support. The majority of infants did not need high ventilatory settings at the moment of pneumothorax occurrence. Needle aspiration was performed in $11(35 \%)$ cases: in all 10 infants with tension pneumothorax and in one case of non-tension pneumothorax. Twenty (65\%) infants were managed expectantly. High-frequency oscillatory ventilation (HFOV) was used in 13 cases $-50 \%$ of infants who were on MV. In total, 26 newborns with pneumothorax were successfully cared for on MV without chest tube placement.

Conclusions: A selected group of haemodynamically stable mechanically ventilated neonates with pneumothorax could be successfully treated without chest-tube insertion.

\section{KEY WORDS:}

pneumothorax, newborn infants, management without chest-tube placement, respiratory support.

\section{INTRODUCTION}

Pneumothorax is a potentially serious complication of the neonatal period. The incidence of symptomatic pneumothorax in newborns is estimated to be as high as $0.05-0.1 \%$. In term and late preterm neonates, the rate of radiologically confirmed pneumothorax ranges from $1 \%$ to $2 \%$, whereas in very low birth weight infants it can reach $3-9 \%[1,2]$.

An established risk factor for pneumothorax is prematurity, due to higher incidence of lung disease in this population of newborns $[3,4]$. Increased risk of pneu-

\section{ADDRESS FOR CORRESPONDENCE:}

Anna Menshykova, Department of Paediatrics No. 2, Danylo Halytsky Lviv National Medical University, 69 Pekarska St., 79010 Lviv, Ukraine, ORCID: 0000-0001-9596-6091, e-mail: anna.menshikova.ua@gmail.com 
mothorax exists in infants with respiratory distress syndrome (RDS), meconium aspiration syndrome (MAS), pulmonary hypoplasia, and in those who require resuscitation and/or respiratory support after birth [5]. This complication is associated with increased morbidity and a higher risk of death, and it occurs despite the use of surfactant and modern ventilation regimens [6]. Pneumothorax occurrence can lead to increased respiratory distress, hypoxaemia, hypercapnia, hypotension, lung collapse, etc. [7].

There is general agreement that infants with an asymptomatic pneumothorax without underlying pulmonary disease do not need treatment [5]. At the same time, management of symptomatic pneumothorax is not clearly defined in haemodynamically stable newborns undergoing assisted ventilation and with a substantial amount of air leak present on chest radiograph [1]. In such cases the most common and traditionally recommended management is chest-tube insertion; however, this invasive procedure may result in substantial complications. Lung injury, chylothorax, phrenic nerve paralysis, and haemorrhagic pericardial effusion have been reported as complications of chest-tube placement [8-13]. An effective alternative for such a newborn with pneumothorax on mechanical ventilation (MV) could be expectant conservative management that prevents the development of complications associated with chest-tube insertion $[1,5]$. One of the options of such management is the use of high-frequency oscillatory ventilation (HFOV), which can ensure a successful result with this approach [6].

Several recent reports described conservative management for neonates with symptomatic pneumothorax, who were on non-invasive respiratory support or mechanical ventilation $[1,5,6]$. Infants with stable respiratory distress, cardiovascular status, and blood gases on relatively low ventilatory settings may not need chest-tube insertion [5].

Because the optimal method for the treatment of clinically apparent pneumothorax in haemodynamically stable newborns remains controversial, it is important to study the effectiveness of expectant management, which can be safer.

The aim of this study was to identify the clinical features of infants with pneumothorax treated without chesttube insertion.

\section{MATERIAL AND METHODS}

In this case series study, we report clinical features and outcomes of 31 outborn infants with pneumothorax, who were successfully managed without chest-tube insertion. In 11 of them needle aspiration was used, and 20 were cared for with expectant management.

In 2016-2017 39 newborns with pneumothorax were treated in our centre without chest-tube insertion. Eight of them died, but their death was not due to progression of pneumothorax. During the mentioned period of time only six newborns were treated with chest-tube placement. In this article we present the data of 31 infants who were managed without chest-tube insertion and subsequently were successfully discharged.

The final decision on the method of treatment was made by the medical team, based on clinical assessment of each patient. In any case of haemodynamic deterioration due to a large and especially tension pneumothorax, pleural drainage was used.

All infants included in the study had respiratory symptoms or deterioration due to pneumothorax occurrence but were haemodynamically stable and able to maintain a normal level of saturation with respiratory support. In the case of technical feasibility in patients with a diagnosis of pneumothorax, who required MV, HFOV was applied immediately. Initial needle aspiration was performed as an emergency intervention mainly in cases of tension pneumothorax and clinical deterioration in spite of respiratory support.

Standard protocols of respiratory support were applied to all newborns with arterial blood gas assessment and routine monitoring of vitals. The presence of pneumothorax was confirmed by X-ray data in all cases with an appropriate repeated investigation. The indications for emergency chest X-ray were acute deterioration in the infant's respiratory status and other clinical data consistent with pneumothorax occurrence including the results of chest transillumination.

Tension pneumothorax was defined as air trapped in the pleural cavity under positive pressure and displacing the mediastinum and its structures (including the lung) toward the opposite side on the chest X-ray.

Endotracheal MV and continuous positive airway pressure (CPAP) were provided with a Servo-i ventilator (Maquet Medical Systems, Wayne, NJ, USA), and HFOV was provided with a Sensormedics 3100A (CareFusion/ Viasys Healthcare, USA).

During the NICU stay, heart rate, arterial blood pressure, haemoglobin oxygen saturation $\left(\mathrm{SpO}_{2}\right)$, body temperature, and urine output were monitored.

For the infants included in the study we collected the most important perinatal data, characteristics of respiratory support, and morbidity.

The obtained data were analysed with the methods of descriptive statistics, correlation (Spearman), and categorical analysis. The measurements with normal distribution are presented as mean \pm standard deviation, and nonparametric data are presented as median (interquartile range) unless otherwise specified or as median (min-max).

\section{RESULTS}

The mean birth weight of the newborns was 2287.42 $\pm 755.45 \mathrm{~g}$ and gestational age was $34.29 \pm 3.2$ weeks. The majority of infants were preterm (24 - 77\%), but only four $(17 \%)$ of them had very low birth weight (less than 
$1500 \mathrm{~g})$. Surfactant therapy was used in seven $(23 \%)$ cases, and the median age at first dose of surfactant administration was 3.5 (3-16) hours. In eight (26\%) infants pneumothorax was diagnosed at the time of hospitalisation to the neonatal intensive care unit. The majority of infants did not need resuscitation after birth but were treated with CPAP because of respiratory deterioration. Pneumothorax occurred in the median age of two (0-4) days (Table 1).

At the moment of pneumothorax occurrence, eight (26\%) infants were on endotracheal MV, two (7\%) - on non-invasive MV, 19 (61\%) - on CPAP, and two (7\%) term newborns were provided with free-flow oxygen through nasal cannulas. CPAP was the most commonly used type of initial respiratory support. Higher initial fraction of inspired oxygen $\left(\mathrm{FiO}_{2}\right)$ and positive pressure on CPAP were reliably associated with higher incidence of tension pneumothorax $\left(r_{S}=0.53\right.$ and $r_{S}=0.58$, respectively; $p<0.05)$. Two infants, who only needed free-flow oxygen, were term newborns with pneumonia, having mild respiratory disorders. The majority of infants did not need high ventilatory settings at the moment of pneumothorax occurrence (Table 2).

Twenty-three (74\%) infants in total were on non-invasive respiratory support at the time of pneumothorax occurrence, but 18 (78\%) of them were switched to MV as soon as the diagnosis was confirmed (Fig. 1). Hence, 26 newborns (84\%) with pneumothorax in our cohort were successfully managed on MV without chest tube insertion.

All infants had an underlying primary lung disease, mainly pneumonia and/or RDS (Table 3 ). It was not possible to exclude development of pneumonia in preterm newborns with RDS in the majority of cases. Pneumonia was a part of the clinical presentation of neonatal sepsis in two infants (5\%). Only three (27\%) cases of RDS were severe, and eight newborns (73\%) had mild to moderate disease.

Ten (32\%) infants had tension pneumothorax, and in five $(16 \%)$ cases bilateral pneumothorax was diagnosed. Tension pneumothorax was mostly seen in preterm newborns ( $80 \%$ vs. $20 \% ; p=0.81$ ); it was bilateral ( $40 \%$ vs. $5 \%$; $p=0.01)$ and occurred more often in infants who did not require resuscitation at birth $(90 \%$ vs. $10 \% ; p=0.04)$ but developed RDS or pneumonia (70\% vs. $14 \% ; p=0.002)$ and were treated with surfactant (50\% vs. $10 \% ; p=0.01$ ) as well as initially managed on CPAP ( $90 \%$ vs. $57 \% ; p=0.06$ ). The incidence of severe IVH was significantly higher in newborns with tension pneumothorax ( $20 \%$ vs. $0 \%$; $p=0.03$ ).

Needle aspiration was performed in 11 (35\%) cases: in all infants with tension pneumothorax and in one case of non-tension pneumothorax. Repeated needle aspirations were performed in two (6\%) newborns with tension pneumothorax. Twenty infants (65\%) were managed expectantly. HFOV was used in 13 cases $-42 \%$ of all infants and $50 \%$ of infants who were on MV (Figs. 1-3).
TABLE 1. Perinatal data of the patients

\begin{tabular}{|l|c|}
\hline Characteristics & $\begin{array}{c}\text { Infants } \\
(n=31)\end{array}$ \\
\hline Birth weight, $\mathrm{g}^{1}$ & $2287.42(755.45)$ \\
\hline Gestational age, weeks ${ }^{1}$ & $34.29(3.2)$ \\
\hline Preterm ${ }^{2}$ & $24(77)$ \\
\hline Positive pressure ventilation at birth ${ }^{2}$ & $11(35)$ \\
\hline Apgar score at the $1^{\text {st }}$ min $^{3}$ & $7(2-8)$ \\
\hline Apgar score at the $5^{\text {th }}$ min $^{3}$ & $7(3-8)$ \\
\hline Surfactant therapy (at least one dose) ${ }^{2}$ & $7(23)$ \\
\hline $\begin{array}{l}\text { Age at the first surfactant dose } \\
\text { administration, hrs }\end{array}$ & $3.5(3-16)$ \\
\hline $\begin{array}{l}\text { Presence of pneumothorax at the time } \\
\text { of hospital admission }\end{array}$ & $8(26)$ \\
\hline Age at pneumothorax occurrence, hrs $^{3}$ & $2(0-4)$ \\
\hline
\end{tabular}

${ }^{7}$ mean (SD), ${ }^{2}$ number of cases (\%), ${ }^{3}$ median (min-max)

TABLE 2. Ventilatory settings at pneumothorax occurrence

\begin{tabular}{|l|c|c|c|}
\hline Parameter & $\begin{array}{c}\text { MV } \\
(n=8)\end{array}$ & $\begin{array}{c}\text { NV } \\
(n=2)\end{array}$ & $\begin{array}{c}\text { CPAP } \\
(n=19)\end{array}$ \\
\hline $\mathrm{FiO}_{2}(\%)$ & $45(40-100)$ & $45(35-55)$ & $35(21-60)$ \\
\hline $\mathrm{PIP}\left(\mathrm{cm} \mathrm{H}_{2} 0\right)$ & $19(18-28)$ & $19(18-20)$ & - \\
\hline $\mathrm{PEEP}\left(\mathrm{cm} \mathrm{H}_{2} 0\right)$ & $5(0)$ & $6(0)$ & $6(5-8)$ \\
\hline $\begin{array}{l}\text { Ventilation } \\
\text { frequency }(/ \mathrm{min})\end{array}$ & $40(35-60)$ & $27.5(25-30)$ & - \\
\hline
\end{tabular}

Median (min-max); 2 infants had free-flow oxygen; MV - mechanical ventilation, $N V$ - non-invasive ventilation, CPAP - continuous positive airway pressure, FiO ${ }_{2}$ - fraction of inspired oxygen, $P I P$ - peak inspiratory pressure, PEEP - positive end-expiratory pressure

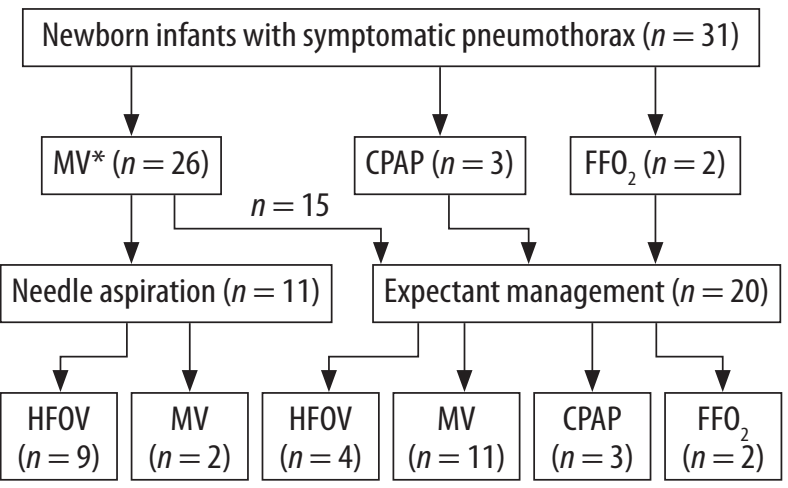

*Infants on mechanical ventilation (MV) before $(n=8)$ and immediately after $(n=18)$ the diagnosis of pneumothorax was conformed; CPAP - continuous positive airway pressure, $\mathrm{FFO}_{2}$ - free-flow oxygen, HFOV - high-frequency oscillatory ventilation

FIGURE 1. Flowchart of the study infants

The incidence of intraventricular haemorrhages was $23 \%$ (seven cases); severe haemorrhages occurred in two newborns (7\%) with tension pneumothorax. Duration of the initial period of endotracheal MV was $66.42 \pm 39.5$ hours and total duration of MV was $81.19 \pm 42.44$ hours. The length of NICU stay was $7.68 \pm 3.99$ days, and the total length of hospital stay was $28.26 \pm 13.31$ days. 
TABLE 3. Primary lung morbidity

\begin{tabular}{|l|c|}
\hline Diseases & $\begin{array}{c}\text { Infants } \\
(n=31)\end{array}$ \\
\hline RDS with possible pneumonia & $10(32)$ \\
\hline RDS & $1(3)$ \\
\hline Pneumonia (aspiration or congenital) & $19(61)$ \\
\hline Pulmonary hypoplasia & $1(3)$ \\
\hline
\end{tabular}

Number of cases (\%); RDS- respiratory distress syndrome
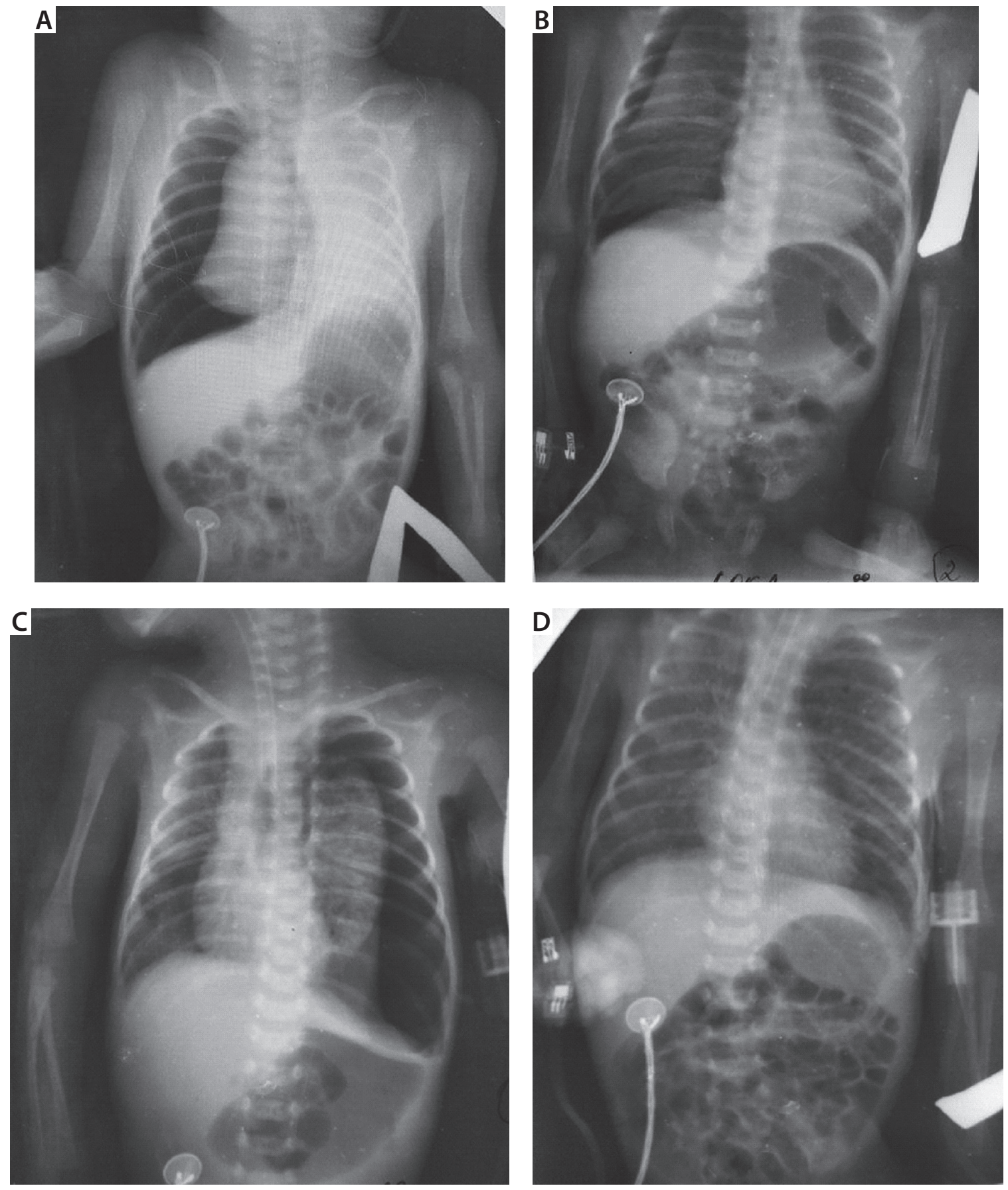

FIGURE 2. A) Chest X-ray of a preterm infant (gestational age - 31 weeks) with right tension pneumothorax. High-frequency oscillatory ventilation (HFOV) was used, and needle aspiration was performed twice. B) Chest X-ray 24 hours later showing improvement in the right tension pneumothorax. C) 48 hours later there was a small amount of air on the right side, but left tension pneumothorax occurred. The infant continued to be on HFOV, and needle aspiration was performed on the left side. D) Resolution of the pneumothoraxes in 96 hours after right tension pneumothorax had occurred and in 48 hours after left tension pneumothorax had occurred 

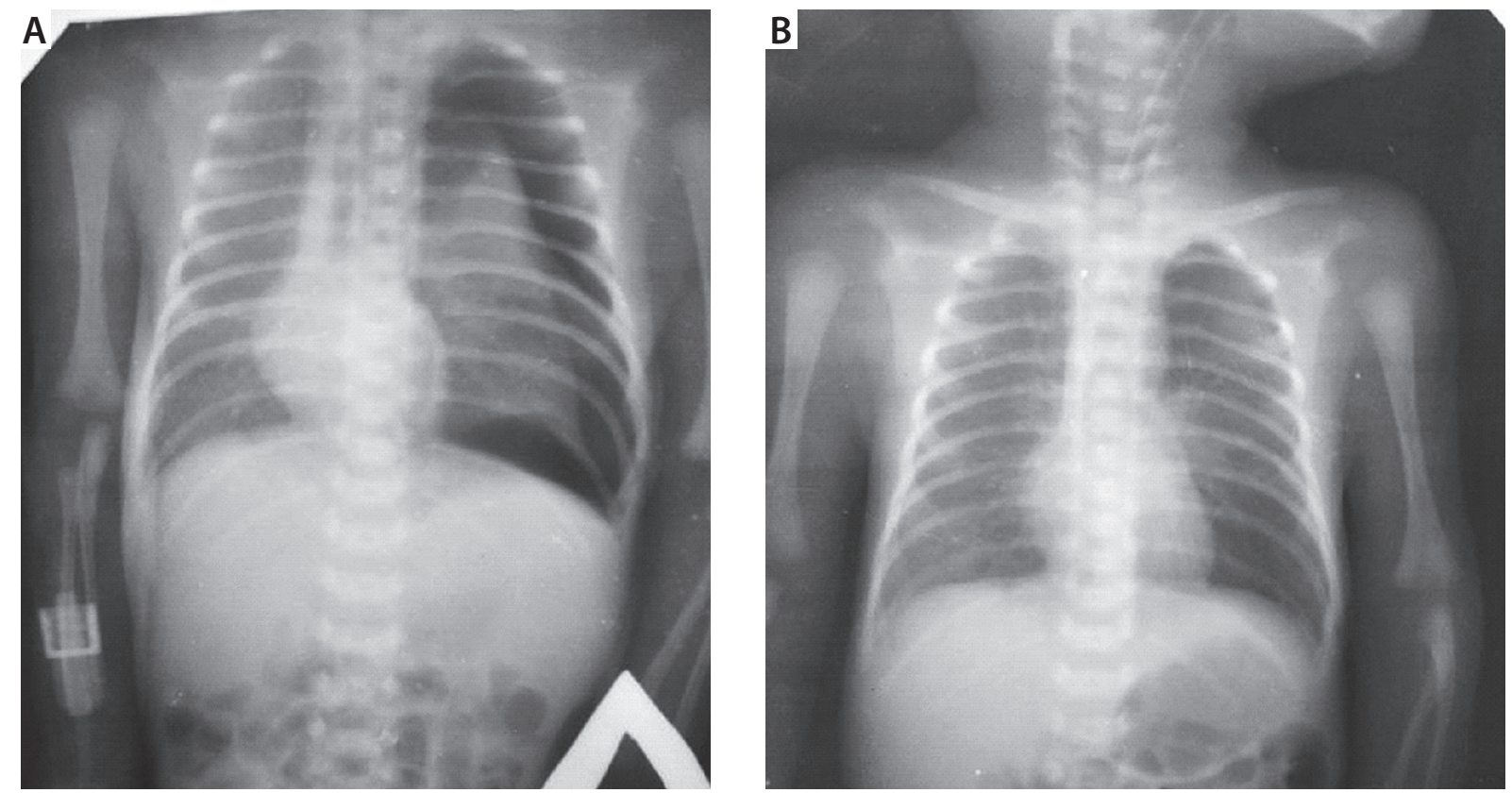

FIGURE 3. A) Chest X-ray of a preterm infant (gestational age - 30 weeks) with a left tension pneumothorax. High-frequency oscillatory ventilation was used, and needle aspiration was performed twice. B) Resolution of the pneumothorax 24 hours later

on the efficacy and safety of less invasive or expectant management of pneumothorax in newborns undergoing assisted ventilation.

The Litmanovitz and Carlo study [5] demonstrated successful treatment of newborns on MV with symptomatic pneumothorax with expectant management. But they avoided chest-tube placement in only $26 \%$ of 136 newborns on MV. In our study 26 (84\%) infants were successfully managed on MV without this intervention. Also, in the aforementioned largest study, it was found that the need for a subsequent chest-tube insertion was higher after needle (catheter) aspiration than with the use of conservative treatment alone. The authors linked it to a possibility of lung injury during the puncture, which could result in an increase in the amount of free air in the pleural cavity [5]. In our study, needle aspiration was performed in all cases of tension pneumothorax which, however, did not lead to chest tube placement. Another study also showed the success and safety of expectant management of pneumothorax in newborns who needed respiratory support (CPAP or MV) [1].

In the above-mentioned studies, infants who did not require chest-tube placement had lower ventilatory settings and better blood gas parameters at the time of pneumothorax diagnosis; they were more mature as well $[1,5]$. In our study the mean GA was $34.29 \pm 3.2$ weeks, and infants did not need high ventilatory settings at the time of pneumothorax occurrence. However, before the onset of pneumothorax, the majority of infants (74\%) were on non-invasive respiratory support (CPAP or NIV), and 18 of them (78\%) were intubated only after pneumothorax occurrence.

One of the options of expectant management of pneumothorax is the application of HFOV. In animal studies, it has been shown that short inspiratory time and small tidal volume, which is created with high frequency ventilation, contribute to rapid reduction in air leak from the affected lungs [15]. Also, at least two clinical trials have shown the efficacy of HFOV application in newborns with pneumothorax $[6,16]$. HFOV provides better gas exchange in the lungs and shorter time of MV administration in newborns with pneumothorax compared to standard MV [17]. In our study, HFOV was used in 50\% of infants, who needed MV, and provided resolution of the pneumothorax without a chest tube insertion. Taking into account that this group included infants with tension pneumothorax, who avoided chest-tube placement, there are reasons to consider the need for early application of this method of respiratory support in the treatment of newborns with pneumothorax.

It must be noted that in the above-mentioned studies, expectant management of pneumothorax treatment was used in newborns with stable haemodynamics, who did not have bradycardia and/or arterial hypotension.

Our study has limitations connected to its observational nature, lack of control group, and relatively small number of patients. At the same time, our data do suggest that haemodynamically stable newborns with clinically apparent and even tension pneumothorax could be successfully managed without chest-tube insertion, especially with timely application of HFOV and/or needle aspiration.

In summary, pneumothorax, which could be managed without chest-tube placement, was most commonly seen in late preterm infants with pneumonia/RDS initially treated with CPAP. The majority of them needed endotracheal intubation and mechanical ventilation to maintain oxygen saturation and other vital functions after pneu- 
mothorax occurrence. Needle aspiration was performed in all newborns with tension pneumothorax diagnosed with X-ray data. Twenty-six newborns with pneumothorax were successfully cared for on mechanical ventilation without chest-tube placement, which demonstrates the feasibility, effectiveness, and safety of such an approach to pneumothorax management in haemodynamically stable newborns. However, a large-scaled randomised trial to prove existent observational data is still needed.

\section{CONCLUSIONS}

A selected group of haemodynamically stable mechanically ventilated neonates with pneumothorax could be successfully treated without chest-tube insertion.

\section{DISCLOSURE}

The authors declare no conflict of interest.

\section{REFERENCES}

1. Kitsommart R, Martins B, Bottino MN, Sant'Anna GM. Expectant management of pneumothorax in preterm infants receiving assisted ventilation: report of 4 cases and review of the literature. Respir Care 2012; 57: 789-793.

2. Duonga HH, Mireab L, Shahb PS, et al. Pneumothorax in neonates: trends, predictors and outcomes. J Neonatal Perinatal Med 2014; 7 : 29-38.

3. Kitsommart R, Janes M, Mahajan V, et al. Outcomes of late-preterm infants: a retrospective, single-center, Canadian study. Clin Pediatr 2009; 48: 844-850.

4. Bhatia R, Davis PG, Doyle LW. Identification of pneumothorax in very preterm infants. J Pediatr 2011; 159: 115-120.

5. Litmanovitz I, Carlo WA. Expectant management of pneumothorax in ventilated neonates. Pediatrics 2008; 122: 975-979.

6. Aurilia C, Ricci C, Tana M, et al. Management of pneumothorax in hemodynamically stable preterm infants using high frequency oscillatory ventilation: report of five cases. Ital J Pediatr 2017; 43: 114-119.

7. Smith J, Schumacher RE, Donn SM, et al. Clinical course of symptomatic spontaneous pneumothorax in term and late preterm newborns: report from large cohort. Am J Perinatol 2011; 28: 163-167.

8. Brooker RW, Booth GR, DeMelto DE, et al. Unsuspected transaction of lung by pigtail catheter in a premature infant. J Perinatol 2007; 27: 190-192.

9. Fraser RS. Lung perforation complicating tube thoracostomy: pathologic description of three cases. Hum Pathol 1988; 19: 518-523.

10. Arya H, William J, Ponsford SN, et al. Neonatal diaphragmatic paralysis caused by chest drains. Arch Dis Child 1991; 66: 441-442.

11. Odita JC, Khan AS, Dincsoy M. Neonatal phrenic nerve paralysis resulting from intercostal drainage of pneumothorax. Pediatr Radiol 1992; 22: 379-381.

12. Kumar SP, Belik J. Chylothorax: a complication of chest tube placement in a neonate. Crit Care Med 1984; 12: 411-412.

13. Quak J, Szatmari A, van den Anker J. Cardiac tamponade in a preterm neonate secondary to a chest tube. Acta Paediatr 1993; 82: 490-491.
14. Walker MW, Shoemaker M, Riddle K, et al. Clinical process improvement: reduction of pneumothorax and mortality in high-risk preterm infants. J Perinatol 2002; 22: 641-645.

15. Ellsbury DL, Klein JM, Segar JL. Optimization of high-frequency oscillatory ventilation for the treatment of experimental pneumothorax. Crit Care Med 2002; 30: 1131-1135.

16. Gonzalez F, Harris T, Black P, et al. Decreased gas flow through pneumothoraces in neonates receiving high-frequency jet versus conventional ventilation. J Paediatr 1987; 110: 464-466.

17. Chen D, Huang XL, Li XP. Clinical application of high-frequency oscillatory ventilation for the treatment of neonatal pneumothorax. Chin J Contemp Pediatr 2012; 14: 499-501. 